\title{
THE NOVEMBER MEETING IN MILWAUKEE
}

The 520th meeting of the American Mathematical Society was held at the Milwaukee Branch of the University of Wisconsin on Friday, November 25, and at Marquette University on Saturday, November 26. There were 130 registrations of which 107 were members of the Society. The sessions began at 9:00 A.M. Friday and ended at noon on Saturday.

The Committee to Select Hour Speakers for Western Sectional Meetings had invited Professor E. H. Spanier of the University of Chicago to address the Society. Professor S. S. Chern presided at the lecture, Aspects of duality in homotopy theory, which was presented on Friday morning at 11:00 A.M.

Presiding officers at the sessions for contributive papers were Professors W. T. Reid, Morris Marden, and H. P. Pettit.

There was a tea for members of the Society and their guests on Friday afternoon at the Milwaukee Branch of the University of Wisconsin, and on Saturday Marquette University played host at a luncheon celebrating the 75th anniversary of their founding. On Friday evening the Society were guests of the Miller Brewing Company for a tour of the brewery followed by a buffet supper and beer at the Miller High Life Inn. The occasion was probably unique in the long and distinguished annals of the Society.

Abstracts of papers presented at the meeting follow. Those with " $t$ " after the abstract number were presented by title. In the case of joint papers, the name of the author who read the paper is followed by (p). Mr. Ornstein was introduced by Professor Irving Kaplansky and Professor L. V. Toralballa by Professor J. W. T. Youngs.

\section{Algebra ANd Theory of Numbers}

123t. K. T. Chen: Integration of paths-geometric invariants and a generalized Baker-Hausdorff formula.

Let the path $\alpha$ in the affine $m$-space $R^{m}$ be given by $x_{i}=\alpha_{i}(t), i=1, \cdots, m$, $a \leqq t \leqq b$. Starting from the line integral $\int_{\alpha} d(i)=\int_{\alpha} d x_{i}$, one defines inductively, for $p \geqq 2, \int_{\alpha} d\left(i_{1}, \cdots, i_{p}\right)=\int_{a}^{b}\left[\int_{\alpha^{t}} t\left(i_{1}, \cdots, i_{p-1}\right)\right] d \alpha_{i_{p}}(t)$ where $\alpha^{t}$ denotes the portion of $\alpha$ with the parameter ranging from $a$ to $t$. The integral remains unchanged when $\alpha$ undergoes a translation. It is further observed that the totality of $\int_{\alpha} d\left(i_{1}, \cdots, i_{p}\right)$, with $p$ fixed, forms a $p$ th order tensor associated with $\alpha$ under linear transformations of $R^{m}$. If all $\int_{\alpha} d\left(i_{1}, \cdots, i_{p}\right)=0$ for $p \leqq r$, then $\alpha$ is said to be of lower central class $r$, which is an affine invariant of $\alpha$. The paths of lower central class 1 are characterized by being closed; and those of lower central class 2 , by enclosing zero algebraic area when projected on any plane in $R^{m}$. Let $X_{1}, \cdots, X_{m}$ be noncommutative indeterminates. Define $\theta(\alpha)=1+\sum \int_{\alpha} d(i) X_{i}+\sum \int_{\alpha} d(i, j) X_{i} X_{i}+\sum \int_{\alpha} d(i, j, k) X_{i} X_{j} X_{h}$ 
$+\cdots, i, j, k, \cdots=1, \cdots, m$. Obtain $d \log \theta\left(\alpha^{t}\right) / d t$ from $\log \theta\left(\alpha^{t}\right)$ by differentiating each of the coefficients of the formal power series $\log \theta\left(\alpha^{t}\right)$ with respect to $t$. By expressing $d \log \theta\left(\alpha^{t}\right) / d t$ in terms of $\sum\left(d \alpha_{i} / d t\right) X_{i}$ and $\log \theta\left(\alpha^{t}\right)$, one proves that $\log \theta(\alpha)$ is a Lie element. As a corollary follows the Baker-Hausdorff formula. (Received October 10,1955.)

\section{N. J. Divinsky: Commutative subdirectly irreducible rings.}

It is shown that a commutative subdirectly irreducible ring is either $(\alpha)$ a field; $(\beta)$ a ring bound to its maximal nilideal with every element being a divisor of zero, and when either the descending or the ascending chain condition is present, it is nilpotent; $(\gamma)$ a ring bound to its maximal nilideal, possessing elements which are not divisors of zero, and possessing a unity element when either the descending or the ascending chain condition is present. An example of a ring of type $(\gamma)$ is given which does not possess a unity element, and an unpublished result of McCoy's is included which proves that every subdirectly irreducible ring (not necessarily commutative) without a unity element is contained in one with a unity. The important consequence of these considerations is the fact that every commutative ring is isomorphic to a subdirect sum of fields, and rings bound to their maximal nilideals. (Received October 11, 1955.)

\section{5t. Franklin Haimo: Endomorphisms of some dihedral groups.}

Let $H$ be an abelian group in which the torsion subgroup $P$ is a direct summand. Let $G$ be a semi-direct product of $H$ and $I_{2}$, the group of two elements, which forms the obvious generalization of the dihedral groups. The endomorphisms and the automorphisms of $G$ are examined. The endomorphisms fall into eight classes. One of these classes has the two-sided ideal property with respect to another class which is closed under endomorphism composition. The holomorph of $H$ can be injected into the automorphism group of $G$. (Received October 13, 1955.)

126t. Melvin Henriksen: On the equivalence of the ring, lattice, and semigroup of continuous functions.

Let $C(X), L(X), S(X)$ denote respectively the ring, lattice, and multiplicative semigroup of all continuous real-valued functions on a topological space $X$. Then, for any topological spaces $X, Y$, the following are equivalent: (i) $C(X)$ and $C(Y)$ are isomorphic. (ii) $L(X)$ and $L(Y)$ are isomorphic. (iii) $S(X)$ and $S(Y)$ are isomorphic. The proof follows in a straightforward way from a theorem of $T$. Shirota (Osaka Math. J. vol. 4 (1952) pp. 121-132). (The author was supported by the National Science Foundation, contract no. NSF-G1129.) (Received October 13, 1955.)

\section{Erwin Kleinfeld: The structure of standard rings.}

In his paper Power-associative rings (Trans. Amer. Math. Soc. vol. 64 (1948) pp. 552-593) Albert introduced a class of rings called standard. The defining relations of such rings are (1) $(w x, y, z)+(x z, y, w)+(w z, y, x)=0$, and (2) $(x, y, z)+(y, z, x)$ $-(y, x, z)=0$, where $(a, b, c)$ denotes $(a b) c-a(b c)$. Interchange $w$ and $x$ in (1) and subtract from (1) in order to obtain the identity (3) $(w x-x w, y, z)=0$. In the present paper the author considers a class of rings more general than standard rings, namely those subject to (2) and (3). For these he obtains structure theorems. The principal result is that simple rings satisfy (2) and (3) if and only if they are either associative or commutative rings. This generalizes Albert's result that simple, finite-dimensional 
algebras are standard if and only if they are either associative or Jordan algebras. (Received August 30, 1955.)

\section{Donald Ornstein: Splittability and modularity in dual vector spaces.}

The author considers two properties of dual vector spaces over arbitrary division rings, splittability and modularity. If the sum of two closed subspaces is closed the space is modular and if every closed subspace admits a closed complement such that the annihilators span the dual the space is splittable. If one space has either property so does its dual. If every subspace of $E$ is closed then obviously $E$ is modular. A sort of converse is proved: if a pair $E, F$ of dual vector spaces is modular then in one space every subspace of countable dimension is closed. With separability conditions the converse is proved. Examples are given of dual vector spaces in which both spaces have nonclosed subspaces. A typical result about splittability is that the direct sum of two splittable spaces need not be splittable. (Received October 13, 1955.)

\section{A. M. Yaqub: On the theory of ring-logics.}

Boolean rings and Boolean logics (=Boolean algebras) are equationally interdefinable in a familiar way (Stone, Trans. Amer. Math. Soc. vol. 40 (1936) pp. 37-111). The theory of ring-logics (Foster, University of California Publications vol. 1 (1951) pp. 385-396) raises this interdefinability and the entire Boolean theory to a more general level. Here a ring $R$ is studied modulo $K$, where $K$ is an arbitrary transformation group in $R$. The Boolean theory results on choosing $K=C=$ "Boolean group," generated by $x^{*}=1-x$ (order $2, x^{* *}=x$ ). More generally in a commutative ring with identity the natural group $N$, generated by $x^{-}=1+x$ (with $x^{2}=x-1$ as inverse) is of particular interest. Thus specialized to $N$, a commutative ring with identity, $(R, \times$, + ) is essentially a ring-logic, $\bmod N$, if the + of the ring is equationally definable in terms of its $N$-logic $(R, \times, \widehat{-}$,$) . It was shown (Foster, loc. cit.) that p$-rings are ringlogics $\bmod N ; p^{k}$-rings were also treated (Foster, Ann. Scu. Norm. Pisa vol. 5 (1951) pp. 279-300). In the present paper it is shown that (A) $p^{k}$-rings, (B) periodic rings, (C) the rings of residues mod $n$ ( $n$ arbitrary) and others are all ring-logics mod $N$. The proofs use some elementary number-theoretic results, notably Fermat-Euler's Theorem. (Received July 14, 1955.)

\section{ANALysis}

130t. Stefan Bergman: An operator generating solutions of a class of partial differential equations.

In Trans. Amer. Math. Soc. vol. 68 (1950) pp. $461 \mathrm{ff}$., operators transforming functions of two complex variables into solutions of certain differential equations in three variables are considered. In the present paper an operator $\mathrm{P}$ is introduced which transforms expressions $X^{m} g(Z)$ into (complex) solutions $\Phi$ of the equation $\mathrm{L}(\Phi) \equiv \Delta_{3} \Phi$ $+F \Phi \equiv \Phi_{X X}-\Phi_{Z Z^{*}}+F\left(Z, Z^{*}\right) \Phi=0$. Here $g(Z)$ is an entire function of the complex variable $Z$, while $F\left(Z, Z^{*}\right)$ is an entire function of $Z$ and $Z^{*}$. The operator $\mathrm{P}$ is defined as follows: Let $q^{(m, m-2 s, s+p)},\left(Z, Z^{*}\right)\left[q^{(m, m-2 s, 8+p)}(Z, 0)=0\right.$, except for $\left.s=p=0\right], m=0,1$, $2, \cdots$, be functions which satisfy the following recursive relations: $q^{(m, m, 0)}=1$,

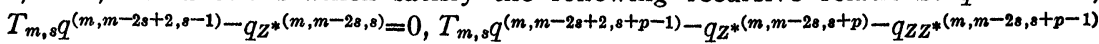
$+F q^{(m, m-2 s, 8+p-1)}=0, s=0,1,2, \cdots,[m / 2] ; p=1,2, \cdots ; T_{m 0}=0, T_{m, 8}=(m-2 s+2)$ - $(m-2 s+1) ;[m / 2]=m / 2$ for an even $m,=(m-1) / 2$ for an odd $m$. Let $\psi^{(m, m)}=g(Z)$ 
$+\sum_{p=1}^{\infty} q^{(m, m, p)}\left(Z, Z^{*}\right) J_{p}(Z ; g) ; \psi^{(m, m-2 s)}=\sum_{p=0}^{\infty} q^{(m, m-2 s, a+p)}\left(Z, Z^{*}\right) J_{s+p}(Z ; g) ;$ $J_{m}\left(Z_{;}^{p-1} g\right)=\int_{0}^{\infty} \cdots \int_{0}^{Z_{m-1}} g\left(Z_{m}\right) d Z_{m} \cdots d Z_{1}$. Then $\Phi\left(X, Z, Z^{*}\right)=\mathrm{P}\left[X^{m} g(Z)\right]$ $\equiv \sum_{:=0}^{[m / 2]} X^{m-28} \psi\left(Z, Z^{*}\right)^{(m, m-2 \theta)}$ are solutions of $\mathrm{L}(\Phi)=0$. They possess the property that $\Phi(X, Z, 0)=X^{m} g(Z), \Phi\left(X, 0, Z^{*}\right)=$ const $X^{m}$. (Received November 21, 1955.)

131t. H. D. Brunk, G. M. Ewing, and W. R. Utz, Jr.: Some Helly theorems for monotone functions.

The principal results of this paper are Helly theorems on the convergence of monotone functions of $n$ real variables. A monotone function, in this paper, means a function monotone in its $n$ individual variables and having all second differences nonnegative or non-positive. A typical theorem asserts that if $\left\{F_{q}\left(x_{1}, x_{2}, \cdots, x_{n}\right)\right\}$ is a sequence of monotone functions on an $n$-dimensional rectangle $S$ and if $A$ is a positive number such that $\left|F_{q}\left(x_{1}, x_{2}, \cdots, x_{2}\right)\right| \leqq A$ for $q=0,1,2, \cdots$, then there exists a sequence of integers $q_{0}<q_{1}<q_{2}<\cdots$ and a monotone function $F\left(x_{1}, x_{2}, \cdots, x_{n}\right)$ bounded by $\pm A$ such that $\lim _{i \rightarrow \infty} F_{q_{i}}\left(x_{1}, x_{2}, \cdots, x_{n}\right)=F\left(x_{1}, x_{2}, \cdots, x_{n}\right)$ on $S$. In proving such theorems it is convenient to first discover limitations on the discontinuities of monotone functions. Such limitations are also discovered for functions monotone in their $n$ variables (but without a condition involving second, or higher, differences) and it is shown that for such functions a Helly theorem analogous to the one quoted above is impossible. (Received October 10,1955.)

132t. Evelyn Frank: $A$ new class of continued fraction expansions for the ratios of generalizations of hypergeometric functions. Preliminary report.

A new class of continued fraction expansions for the ratios of two generalizations of hypergeometric functions is described in detail. A number of such ratios are expanded, and the convergence of the corresponding continued fractions is determined as well as the functions to which the continued fractions converge in various regions. Recurrence relations between the numerators and denominators of the approximants of these continued fractions are found. Many special relations are obtained between functions, finite continued fractions, and infinite continued fractions. Specialized continued fraction expansions and specialized functions are discussed, and integral representations for such continued fractions are found. (Received October 6, 1955.)

133. R. E. Fullerton: The Cesari inequality for surfaces defined over a two-dimensional manifold.

Let $M$ be a compact two-dimensional metric manifold with boundary and $S$ a Fréchet surface defined over $M$. Let $T$ be a representation of $S$ and let $f$ be a function defined on $E_{3}$ having the property that for any two points $p_{1}, p_{2}$ in $E_{3},\left|f\left(p_{1}\right)-f\left(p_{2}\right)\right|$ $\leqq K\left|p_{1}-p_{2}\right|$. Let $D^{-}(t)$ be the subset of $M$ defined by $f(T(p))<t$ for $p \in[S]$ and let $l(t)$ be the length of the boundary of $D^{-}(t)$ defined in a generalized sense by use of prime ends in a manner similar to that used by Cesari [Surface area, Annals of Mathematics Studies, Princeton, to appear]. It is shown that $l(t)$ is an integrable function over $(-\infty, \infty)$ and that if $L(S)$ is the Lebesgue area of $S$ then $K L(S) \geqq \int_{-\infty}^{\infty} l(t) d t$. This generalizes a similar theorem of Cesari [loc. cit.] for the case in which $M$ is a simply connected plane set. (Received October 10, 1955.)

134. F. W. Gehring: The converse of Fatou's theorem for positive harmonic functions.

Suppose that $u(z)=u(x, y)$ is non-negative and harmonic in the upper half plane 
$y>0$ and let $\partial u(z) / \partial \xi_{d}=\cos d u_{x}(z)+\sin d u_{y}(z)$. A simple extension of the Wiener tauberian theorem leads to very direct and elementary proofs for results of which the following are special cases. THEOREM 1: Suppose that $0<a, b<\pi$, that $a \neq b$, and that $u\left(t e^{i a}\right) \rightarrow A$ and $u\left(t e^{i b}\right) \rightarrow B$ as $t \rightarrow 0+$. Then for each $0<c<\pi$ and each $d, t\left(\partial u / \partial \xi_{d}\right)$ $\cdot\left(t e^{i c}\right) \rightarrow C \sin (d-c)$ as $t \rightarrow 0+$ where $C=(B-A) /(b-a)$. THEOREM 2: Suppose that $0<a, c<\pi$, that $c \neq \equiv d \bmod \pi$, and that $u\left(t e^{i a}\right) \rightarrow A$ and $t\left(\partial u / \partial \xi_{d}\right)\left(t e^{i c}\right) \rightarrow C \sin (d-c)$ as $t \rightarrow 0+$. Then for each $0<b<\pi, u\left(t e^{i b}\right) \rightarrow B$ as $t \rightarrow 0+$ where $B=A+C(b-a)$. Next suppose that $f(z)=u(z)+i v(z)$ is bounded and analytic in the upper half-plane $y>0$. From Theorem 2 and a well known tauberian theorem we can immediately obtain the following variant of a result due to Lindelöf. THEOREM 3: Suppose that $0<a$, $b<\pi$ and that $u\left(t e^{i a}\right) \rightarrow A$ and $v\left(t e^{i b}\right) \rightarrow B$ as $t \rightarrow 0+$. Then for each $0<c<\pi, f\left(t e^{i c}\right) \rightarrow A+i B$ as $t \rightarrow 0+$. (Received October 13, 1955.)

\section{P. E. Guenther: Formulation of a Riemann problem.}

A Riemann problem for a linear homogeneous $q$-difference system $Y(q x)$ $=A(x) Y(x), x, q$ complex, $|q|>1$, with elements of $A(x)$ rational functions of $x$ was stated and solved by G. D. Birkhoff in the case that the related characteristic equation has finite nonzero roots and that $Y(x)$ is free from logarithmic terms (Proceedings of the American Academy of Arts and Sciences vol. 49 (1913) pp. 559-568). The present note considers the system of order two after reduction to the canonical form developed by G. D. Birkhoff and the author (Proc. Nat. Acad. Sci. U.S.A. vol. 27 (1941) pp. 218-222). For systems of order two there are three distinct canonical forms, one of which corresponds to the case mentioned above for which the Riemann problem has been solved. As a first step in formulating a Riemann problem for the remaining cases the behavior of the solution $Y_{0}(x)$ about zero and $Y_{\infty}(x)$ about infinity is shown to depend upon the corresponding behavior of the solution of a typical summation problem. For certain types of such typical solutions the characteristic constants involved in the representation of the $q$-periodic elements of $P(x)$ in the equation $Y_{0}(x)=Y_{\infty}(x) P(x)$ are determined. (Received October 11, 1955.)

136. D. L. Guy: Marcinkiewicz theorem for Hankel transforms. Preliminary report.

Let $-1 / 2<\alpha<1 / 2$, and $\mathfrak{M}_{\alpha}(f)^{2}=\int_{0}^{\infty} f(x)^{2} x^{2 \alpha}\left(1+x^{2 \alpha}\right)^{-1} d x$. Let $\mathfrak{M}_{\alpha}$ be the class of $f$ for which $\mathfrak{M}_{\alpha}(f)^{2}<\infty$. Given $k(x, t)$, let $K(f ; t)=\int_{0}^{\infty} f(x) k(x, t) d t$. Let $\bar{f}_{\nu}(t)$ be the Hankel transform of $f(x)$ of order $\nu \geqq-1 / 2$. If $g_{\nu}(t)=\psi(t) f_{\nu}(t)$, let $\Psi^{(\nu)}(f(x))=g(x)$. Finally, $\psi(t) \in M_{N}(C)$ if $\psi(t)$ is bounded by $C$ and the variation of $\psi(t)$ on $\left[2^{n-1}, 2^{n}\right]$ is no more than $C$ for all $n \geqq N$. Theorem 1 . If $k(x, t)$ is bounded and $K$ is a bounded transformation of $L^{2}$ into $L^{2}$, and if $f \in \mathfrak{M}_{\alpha}, 0<\alpha<1 / 2$, then $\int_{0}^{\infty} K(f ; t)^{2}(1+t)^{-2 \alpha} d t$ $\leqq A_{\alpha} \mathfrak{M}_{\alpha}(f)^{2}$ [Hirschman, $A$ note on orthogonal systems, to appear in Pacific Journal of Mathematics]. Theorem 2. If $f \in \mathfrak{M}_{\alpha}, 0 \leqq \alpha<1 / 2$, then there are positive constants $A^{\prime}(\alpha, \nu), A^{\prime \prime}(\alpha, \nu)$, such that $A^{\prime} \leqq \int_{0}^{\infty} \int_{0}^{\infty}\left[f_{\nu}(t) t^{-\nu-1 / 2}\right]^{2} Q_{\alpha, \nu}(t, u) d t d u / \mathfrak{M}_{\alpha}(f)^{2} \leqq A^{\prime \prime}$ where $Q_{\alpha, \nu}(t, u)=\lim _{\epsilon \rightarrow 0+} \int_{1}^{\infty} \int_{0}^{\infty} \exp \left(\epsilon^{2}-x^{2}\right) x^{1-\nu} J_{\nu}(x t) J_{\nu}(x u) J_{\nu}(x a) a^{-\nu-1-2 \alpha} d x d a$ [Hirschman, Weighted quadratic norms, etc., to appear in Canadian Journal of Mathematics]. Theorem 3. If $\psi(t) \in M_{N}(C)$ and $f(x) \in \mathfrak{M}_{\alpha}, 1<1 / 2<\alpha<1 / 2$, then $\mathfrak{M}_{\alpha}\left(\Psi^{(\nu)}(f(x))\right)$ $\leqq A \mathfrak{M}_{\alpha}(f(x))$, where $A$ depends only on $\alpha, \nu$, and $N$ [Hirschman, Ibid.; Marcinkiewicz, Studia Math. vol. 8, p. 78]. (Received September 20, 1955.)

137t. W. T. Guy, Jr.: On a Mathieu integral transform. II.

A Mathieu integral transform is defined using a Mathieu function as the kernel. 
A short table of properties of this transform and transforms of some special functions are developed. (Received October 13, 1955.)

138. W. B. Jurkat and Alexander Peyerimhoff (p): On a Tauberian theorem for resultants.

The paper deals with the following problem stated by P. Erdös: For what numbers $\alpha$ can the relation $s_{n}=n+O\left(n^{\alpha}\right)$ be derived from the relation $\sum_{\nu_{-0}}^{n} s_{n-\nu} a_{\nu}=n^{2} / 2+O(n)$, $a_{\nu} \geqq 0\left(s_{n}=\sum_{\nu_{-0}}^{n} a_{\nu}\right)$. Erdös has shown that $s_{n}=n+o(n)$ holds whereas $s_{n}=n+o\left(n^{1 / 2}\right)$ cannot be derived. V. G. Avakumovic proved that the relation $s_{n}=n+O\left(n^{3^{1 / 2}-1+e}\right)$ holds for any $\epsilon>0$. The paper gives a proof of the relation $s_{n}=n+O\left(n^{2 / 3} \log n^{1 / 3}\right)$. (Received October 13, 1955.)

139. E. G. Kimme: A Taylor expansion for a certain class of functionals.

Let $\Sigma$ be the set of real functions of bounded variation on the whole real line, continuous from the right and vanishing at $-\infty$. Let $h_{s}(t)$ denote that element of $\Sigma$ which is zero for $t<s$ and one for $t \geqq s$. If $F$ is a functional of $x(t)$ in $\Sigma$, define $D_{s} F[x]$ $=\lim _{\alpha \rightarrow 0}\left(F\left[x+\alpha h_{s}\right]-F[x]\right) / \alpha$ if the limit exists. Repeated "derivatives" are defined in the usual way: $D_{s_{1} s_{2}} F[x]=D_{s_{1}}\left(D_{s_{2}} F[x]\right)$ and so on. Using techniques involving approximations to functions $x \in \Sigma$, it is shown that under certain rather involved continuity hypotheses on $F[x]$ and $D_{s} F[x]$ one has $F[x]=F[0]+\int_{0}^{1} \int_{-\infty}^{1} D_{s} F[\mu x]$ $d x(s) d \mu$. Iteration of this result leads to a finite series with terms of the form $\int^{\infty} \cdots-_{-\infty}(k) \cdots \int_{-\infty}^{\infty} D_{s_{1}} \cdots s_{k} F[0] d x\left(s_{1}\right) \cdots d x\left(s_{k}\right) / k$ ! and a remainder, reminiscent of Taylor's theorem and the classical Volterra extension thereof. A sufficient condition for $F$ to be representable as an infinite series of such terms is exhibited. If $F$ is linear, these results reduce to a version of Riesz's theorem on the representation of linear operators on a Hilbert space. If $F[x]=f\left(x\left(a_{1}\right), \cdots, x\left(a_{N}\right)\right)$ the stated results reduce to the $N$-dimensional Taylor theorem for $f$. (Received October 10,1955.)

140t. Jacob Korevaar: Notes on differential equations. I. Analog of a nonlinear Tauberian theorem of Erdös.

In connection with his proof of the prime number theorem Erdös obtained the following nonlinear Tauberian theorem (J. Ind. Math. Soc. vol. 13 (1949) pp. 131147). When (1) $\sum_{k=1}^{n} a_{k}\left(s_{n-k}+k\right)=n^{2}+O(n)$ and (2) $a_{k} \geqq 0$, then (3) $s_{n}=\sum_{1}^{n} a_{k}$ $=n+O(1)$. Setting $\sum a_{k} e^{-k x}=y(x)$, relation (1) implies that (4) $y^{\prime}-y^{2}=-2 / x^{2}$ $+O(1 / x)$ as $x \downarrow 0$. Question. Do the solutions of (4) satisfy an estimate just as sharp as (3) ? The answer is yes: equation (4) implies that either $y=1 / x+O(1)$ or $y=-2 / x$ $+O(1)$ as $x \downarrow 0$. For the proof one uses the familiar substitution $y=-u^{\prime} / u$ to obtain the linear equation $u^{\prime \prime}+\left\{-2 / x^{2}+O(1 / x)\right\} u=0$. Analogs of well-known methods (Bellman, Stability theory of differential equations, New York, 1953) now show that either $u=c_{1} / x+O(1)$ and $u^{\prime}=-c_{1} / x^{2}+O(1 / x)$ with $c_{1} \neq 0$, or $u=c_{2} x^{2}+O\left(x^{3}\right)$ and $u^{\prime}=2 c_{2} x+O\left(x^{2}\right)$ with $c_{2} \neq 0$, or $u \equiv 0$. This proves the result. It is conjectured that (1) without (2) implies that either (3) holds, or $s_{n}=-2 n+O(1)$. (Received October 3, 1955.)

141t. Karel deLeeuw: On a type of convexity in the space of $n$ complex variables.

Let $C^{n}$ be the space of $n$ complex variables. For $X$ a bounded subset of $C^{n}$ denote by $\widehat{X}$ the set of all $y$ in $C^{n}$ that satisfy for every polynomial $P,|P(y)| \leqq \operatorname{Sup}|P(x)|$ 
where $x$ runs over $X$. Let $S$ be a finitely generated subsemigroup of an abelian group. Denote by Hom $(S, C)$ the set of all multiplicative maps from $S$ into the complex numbers that are one on the unit of $S$ if $S$ contains a unit. Let $x_{1}, \cdots, x_{n}$ be generators of $S$. Imbed Hom $(S, C)$ in $C^{n}$ by identifying $\phi$ in Hom $(S, C)$ with the point $\left(\phi\left(x_{1}\right), \cdots, \phi\left(x_{n}\right)\right)$. The image of $\operatorname{Hom}(S, C)$ is a variety in $C^{n}$ that will be denoted by $V$. Let $T$ be all $\left(z_{1}, \cdots, z_{n}\right)$ in $V$ that satisfy $\left|z_{i}\right| \leqq 1$ for all $i$. $X$ is called $V$-circular if whenever $\left(w_{1}, \cdots, w_{n}\right)$ is in $X$ and $\left(z_{1}, \cdots, z_{n}\right)$ is in $T$, the point $\left(z_{1} w_{1}, \cdots, z_{n} w_{n}\right)$ must be in $X$. Theorem: If $X$ is a subset of $V$, is $V$-circular and contains the point $(1, \cdots, 1)$, then $\widehat{X}$ is the intersection of $V$ and all sets of the form $\left|z_{1}^{a} z_{2}^{b} \cdots\right| \leqq r$ that contain $X$. (Received October 10,1955.)

\section{Karel deLeeuw: On homogeneous Banach algebras. I. Identi-} fication of the maximal ideal space.

Let $G$ be a compact abelian group, $G^{*}$ its dual. A Banach algebra $A$ of continuous functions on $G$ (with multiplication pointwise and topology stronger than pointwise convergence) is a homogeneous algebra if it contains the constant functions, separates points of $G$, is invariant under translations by $G$, and is generated by the semigroup $S_{A}$ of characters that it contains. Let Char $\left(S_{A}\right)$, supplied with the compact-open topology, be the set of all not identically zero homomorphisms of $S_{A}$ into the complex numbers. Since distinct multiplicative functionals on $A$ induce distinct homomorphisms of $S$ into the complex numbers, the maximal ideal space $M(A)$ is imbedded in Char $\left(S_{A}\right)$. Let $\hat{A}$ be the Gelfond representation of $A$. For $\chi \in S_{A}$ let $p(\chi)$ be the norm of $\chi$ in $\hat{A}$. The result of the above abstract is used for THEOREM: $M(A)$ consists of those $\phi \in C$ Char $\left(S_{A}\right)$ satisfying $|\phi(\chi)| \leqq p(\chi)$ for each $\chi \in S_{A}$. THEOREM: Let $S$ be any subsemigroup of $G^{*}$ containing the unit and generating $G^{*}$. Then the compact subsets of Char $(S)$ that can occur as maximal ideal spaces of homogeneous algebras on $G$ are those that contain $\phi \equiv 1$ and are the intersection of sets of the form $\{\phi:|\phi(\chi)| \leqq r$ for fixed $\chi \in S\}$. (Received October 10, 1955.)

\section{3t. L. F. Meyers: Transformations of Hermitian functionals} characterized by an extremum property.

Let $A$ and $B$ be positive definite Hermitian bilinear functionals on a Hilbert space $H$. Consider all linear transformations $T$ of $H$ into itself carrying $A$ into $B$, so that $A(T u, T v)=B(u, v)$ for all $u, v$ in $H$. Loewner [Bull. Amer. Math. Soc. Abstract 52-1-19] showed that among all such transformations, the one differing least from the identity transformation $I$ in the sense of absolute norm based on $A$ or $B$ is, in matrixstyle notation, $+\left(A^{-1} B\right)^{1 / 2}$, under certain conditions. New results are the following. The same minimal transformation is obtained (although without uniqueness) if $T-I$ is to have smallest bound with respect to $A$ or $B$, and $B$ is bounded with respect to $A$. If $A$ and $B$ are joined by a sufficiently smooth curve in the space of positive definite functionals, then a noncommutative product integration of minimizing transformations can be carried out along the curve, an approximation to the integral being $\Pi_{j=1}^{k}\left(C_{j-1}^{-1} C_{j}\right)^{1 / 2}$, where the $C_{i}$ 's are points of the curve. (Received October 10,1955).

\section{W. T. Scott: Linear Baecklund transformations.}

For a linear Baecklund transformation between two sets, $\eta$ and $y$, of $m$ functions in $n(n \geqq 2)$ independent variables, there are determined conditions on the coefficients of the transformation under which the transformation links certain linear systems of 
partial differential equations in $\eta$ and in $y$. The coefficient conditions are shown to be conditions that a certain system be passive in the sense of Riquier. In the case where $n=2$ it is possible to duplicate some results of Loewner for $m=2, n=2$, and to characterize completely the transformation coefficients and the linked systems. In the general case it is shown that linked first order linear systems have the same characteristic equation. In the case where $m=1$ it is shown that the type of linked equations is the same, thus duplicating a result of Resch for $m=1, n=3$. (Received October 13, 1955.)

\section{L. V. Toralballa: On locally distributive algebras.}

A locally distributive algebra is a system $\Sigma(C,+, x)$ satisfying: $1 .(C,+)$ is a real Banach space. 2. $(C, x)$ is closed. $3 . x$ is continuous with respect to both factors, simultaneously. 4. $|a \times b| \leqq|a| \cdot|b| .5 .(\alpha a) \times(\beta b)=(\alpha \beta)(a \times b) .6 .|a+b|^{2}+|a-b|^{2}$ $=2|a|^{2}+2|b|^{2}$. Define the angle $(a, b)$ between two non-zero vectors by $\cos (a, b)$ $=\left(|a|^{2}+|b|^{2}-|a-b|^{2}\right) / 2|a||b|$. Given vectors $a$ and $c$ and a class $B$ of vectors $b$ of the same length, then for every $\epsilon>0$, there exists a $\delta>0$ such that if $(a, b)<\delta$ then $|c(a-b)-(c a-c b)|<\epsilon$. Given vectors $a, c$ then for every $\epsilon>0$ there exists a $\delta>0$ such that if $|a-b|<\delta$ then $|c(a-b)-(c a-c b)|<\epsilon$. If $S$ is a sufficiently smooth rectifiable curve in $\Sigma$ and $f$ is a function on $\Sigma$ to $\Sigma$ continuous over $S$ then $\int_{S} f(z) d z$ exists. (Received October 11, 1956.)

\section{F. M. Wright: Some formulas related to certain $C$-fractions.}

Let $\left\{k_{j}\right\}$ and $\left\{p_{i}\right\}(j=0,1,2, \cdots)$ be given infinite sequences of integers with $k_{0}=1, p_{0}=-1$ and $k_{j} \geqq 0, p_{j} \geqq 0$ for $j=1,2,3, \cdots$. Let $\left\{\alpha_{n}\right\} \quad(n=1,2,3, \cdots)$ be the sequence of integers with $\alpha_{1}=1$ and such that if $h$ is any non-negative integer then: (i) $\alpha_{n}=1$ for $n=2 \cdot \sum_{j=0}^{n}\left(k_{j}+p_{j}\right)+i\left(i=2,3, \cdots, 2 k_{h+1}+1\right)$; (ii) $\alpha_{n}=2$ for $n=2 \cdot \sum_{i=0}^{h}\left(k_{j}+p_{j}\right)+2 k_{h+1}+i\left(i=2,3, \cdots, 2 p_{h+1}+1\right)$. Further conditions imposed on the sequences $\left\{k_{i}\right\},\left\{p_{i}\right\}$ assure that the sequence $\left\{\alpha_{n}\right\}$ is infinite. The author considers here $C$-fractions $K_{(n)}\left[c_{n} w^{\alpha_{n}} / 1\right]$ which are either nonterminating or terminating with $\left[2 \cdot \sum_{j-0}^{m+1}\left(k_{j}+p_{j}\right)+1\right]$ partial quotients, where $m$ is some given non-negative integer. Let $C(w)$ denote the above $C$-fraction or the above $C$-fraction with a partial quotient $c w / 1$ attached according as this $C$-fraction is non-terminating or terminating. Formulas involving the coefficients of $C(w)$ and the coefficients in its corresponding power series $\sum_{n=0}^{\infty} u_{n} w^{n+1}$ corresponding, in the most part, to results discovered by W. T. Scott (Ann. of Math. vol. 51 (1950) pp. 56-67) are obtained in a relatively simple and direct manner. These formulas, which contain determinant formulas for the coefficients $c_{n}$ of $C(w)$ such that $\alpha_{n}=2$, are then used to obtain determinant formulas for the remaining coefficients $c_{n}$ and information concerning the determinants $\Delta_{1, n}$ (Received October 10, 1955.)

\section{Applied Mathematics}

\section{7t. C. C. Elgot: Complex least squares.}

The least square solution of a set of linear equations with complex coefficients is of ten accomplished by applying theorems and techniques expounded by $T$. Banachiewicz to the set of equivalent real equations. Not only are the theorems and the techniques extendible to the complex case, but less than half the number of real operations is required to compute the solution of the normal equations within the complex field rather than within the real field. A useful relationship among the weights of the real unknowns, apparently not previously observed, becomes obvious. The technique in 
the complex case is as readily adaptable to high speed machine computation as in the real case. .(Received October 10,1955.)

148. A. C. Eringen: A transform technique for boundary value problems in 4th order partial differential equations.

Let $u\left(x, \lambda_{n}\right)$ be the eigenfunction of any fourth order linear differential equation $L u+\lambda u=0, L u \equiv u^{(i v)}+\left(p u^{\prime}\right)^{\prime}+q u(a \leqq x \leqq b)$, and four homogeneous uncoupled boundary conditions at two ends $x=a$ and $x=b$. The system is assumed to be selfadjoint. Define $T$-transform by $f_{T}\left(\lambda_{n}\right)=N_{n}^{-2} \int_{a}^{b} f(x) u\left(x, \lambda_{n}\right) d x$ where $N_{n}$ is a normalization factor. One has the inversion theorem: $f(x)=\sum_{n} f_{T}\left(\lambda_{n}\right) u\left(x, \lambda_{n}\right)$. It is shown that $\{L f\}_{T}=\lambda_{n} f_{T}\left(\lambda_{n}\right)+B\left\{f, u\left(x, \lambda_{n}\right)\right\}$ where $B$ contains boundary values. With the use of this result one can solve two-point boundary-value problems concerning inhomogeneous boundary conditions in fourth order partial differential equations. An example is a vibrating beam with time dependent boundary conditions and space dependent initial and final conditions. (Received September 19, 1955.)

149. P. C. Hammer (p) and A. H. Stroud: Numerical integration over simplexes.

In this paper two sets of formulas are given for numerical evaluation of integrals over the $n$-simplex. One formula holds precisely for the general quadratic polynomial and is based on $n+1$ evaluation points. The other formula holds for the cubic polynomial and is based on $n+2$ evaluation points. These formulas are affine invariant. (Received October 11, 1955.)

150t. J. P. Roth: An application of algebraic topology to numerical analysis, IV. Tearing versus partitioning, case histories.

The first example is a 40 branch network of the type used for solving Poisson's equation (Kron. J. of App. Phys. vol. 24 (1953) pp. 965-980). Number of multiplications required for the general solution: by tearing 250, Maxwell's mesh method coupled with (a) partitioning 1170, (b) Gauss elimination 4000. "Multiplying-out" operations were also less for tearing. Storage requirements for the general solution were considerably less, a change in the original data necessitates relatively few changes in the solution. The second example is a 56 branch network with a symmetric impedance tensor. In this example tearing exceeds standard methods even more, but for the third example tearing is useless. (Received October 13, 1955.)

\section{Geometry}

\section{1t. M. M. Day: Some criteria for inner product spaces.}

One result ${ }_{\mathrm{g}}$ given has as a special case the following generalization of results of Day (Trans. Amer. Math. Soc. vol. 62 (1947) pp. 320-337), Schoenberg (Proc. Amer. Math. Soc. vol. 3 (1952) pp. 961-964), and Kasahara (Proc. Japan. Acad. vol. 30 (1955) pp. 846-848). Let $E$ be a normed linear space and let $\sim$ be one of the relations $=, \geqq$, or $\leqq$ (the same one throughout $E$ ). Then any one of the following conditions is necessary and sufficient that $E$ be an inner product space: For each pair $x, y$ of points of $E$ of norm one there is a $\lambda$ depending on $x$ and $y$ such that $0<\lambda<1$ and $1 \sim \lambda(1-\lambda)\|x-y\|^{2}+\|\lambda x+(1-\lambda) y\|^{2}$. Another result extends a characterization of L. M. Blumenthal (Pacific Journal of Mathematics vol. 5 (1955) pp. 161-167). Let $E$ be a complete, externally convex, metric (or a normed linear) space; then $E$ is isometric with an inner product space if and only if $E$ has the queasy euclidean four- 
point property: If $p$ and $r \in E$, there is a $q$ metrically between $p$ and $r$ such that for every $s \in E$ the quadruple $p, q, r, s$ is isometrically embeddable in a euclidean plane. (Received October 10, 1955.)

\section{Statistics and Probability}

\section{G. E. Baxter: $A$ Wiener process distribution of the arc-sine law} type.

Let $\{x(t), 0 \leqq t<\infty\}$ denote the Wiener process, i.e. a Gaussian stochastic process with $E\{x(t)\}=0, E\{x(t) x(s)\}=\min \{s, t\}$, and with $x(t)$ a real-valued continuous function of $t$. Furthermore, let $m\{A\}$ denote the Lebesgue measure of the $t$-set $A$. For any $\epsilon>0$, define the random variable $T(x, \epsilon)$ to be the least upper bound (possibly infinite) of the $T$-set for which $\lim _{t \rightarrow T}-\operatorname{sgn}[x(t)-\epsilon t]=1$. It is shown that the joint probability density of the distribution function $P\{m(t: x(t)>\epsilon t) \leqq u, T(x, \epsilon) \leqq v\}$ is $\left(\epsilon /\left(2 \pi v^{3}\right)^{1 / 2}\right)$ exp $\left\{-\epsilon^{2} v / 2\right\}, v>u \geqq 0$, zero otherwise. The corresponding marginal distributions are of particular interest. $P\{m(t: x(t)>\epsilon t) \leqq u\}=\int_{0}^{u} \int_{w}^{\infty}\left(\epsilon /\left(2 \pi v^{3}\right)^{1 / 2}\right)$ $\cdot \exp \left\{-\epsilon^{2} v / 2\right\} d v d w$ is a result along the line of the famous arc-sine law of Erdös and Kac [Bull. Amer. Math. Soc. vol. 53 (1947) pp. 1011-1020]. On the other hand $P\{T(x, \epsilon) \leqq v\}=\int_{0}^{0}\left(\epsilon /\left(2 \pi w^{3}\right)^{1 / 2}\right)$ exp $\left\{-\epsilon^{2} w / 2\right\} d w$ trivially implies the strong law of large numbers for the Wiener process. (Received October 13, 1955.)

153. R. J. Buehler: Estimation of a product of two binomial parameters by confidence intervals.

The product $P_{1} P_{2}$ of two binomial parameters arises when two elements operate in parallel (either must succeed) or in series (each must succeed). If both elements come from the same population, $P_{1} P_{2}$ becomes $P^{2}$, and (Neyman) confidence intervals for estimation of $P^{2}$ are given by a trivial application of well known formulas for estimating $P$. Otherwise a nuisance parameter appears, and many more-or-less reasonable systems of intervals can be obtained. Conditions leading to uniqueness are studied after specializing to the case of highly reliable parallel elements $\left(P_{1}\right.$ and $\left.P_{2} \ll 1\right)$. The conventional solution for estimating $P$ suggests three conditions, "symmetry," "regularity," and "shortness"; and these eliminate most of the less reasonable solutions. One more condition is added, making the solution unique; and a table of intervals is obtained, based on a Poisson approximation, which is valid when $P_{1}$ and $P_{2} \ll 1$ and the sample sizes are moderate or large. (Received October 6, 1955.)

154. M. Z. Krzywoblocki: On the application of Kampé de Fériet's relation: Hilbert spaces and random functions to turbulence in compressible fluids.

Kampé de Feriet was the first to apply the results of the theory of the random functions to the theory of the homogeneous turbulence. Suppose that we have a probability measure defined on an abstract space $\Omega$; let $T_{t} \omega$ be a group of measure preserving transformations of the space $\Omega$ onto itself, possessing the property $T_{0+t}$ $=T_{s} T_{t}$. Let $f(\omega)$ denote a real measurable function defined on the measure space $(\Omega, S, \mu) ; F(t, \omega)=f\left(T_{t} \omega\right)$ defines a stationary random function of the variable $t$. The harmonic analysis of that process can be obtained by means of the harmonic analysis of the group of unitary transformations of the Hilbert space $L_{2}$ onto itself, defined by $U_{t} g(\omega)=g\left(T_{t} \omega\right)$. Kampe de Fériet applied this relation to the theory of turbulence in an incompressible fluid. The author of the present paper applied this relation to the theory of turbulence in compressible fluids. (Received September 30,1955.) 


\section{J. V. Talacko: Some conditional distributions of the Brownian plane area.}

In the study of Brownian motion from the point of stochastic processes, the Wiener's Gaussian stochastic variables may be used. Consider a Brownian plane area, bounded by an arc and its chord, given in parametric form by $x=X(t), y=Y(t)$; $0 \leqq t \leqq 2 \pi$; where $X(t)=X\left(t_{0}\right)+\xi\left(t-t_{0}\right)^{1 / 2}, t>t_{0}, t$ is a continuous parameter and $\xi$ is the Gaussian random variable. This area may be evaluated by a stochastic integral with an almost sure limit: $A=\sum_{n=1}^{\infty}\left[\xi_{n}\left(\eta_{n}^{\prime}-\eta^{\prime} 2^{1 / 2}\right)-\eta_{n}\left(\xi_{n}^{\prime}-\xi^{\prime} 2^{1 / 2}\right)\right]$ and with the characteristic function: $C_{A}(x ; v, \rho)=\pi v / \sinh \pi v \exp \left\{\rho^{2} / 2-\left(\rho^{2} / 2\right) \pi v \cosh \pi v / \sinh \pi v\right\}$. It is non-Gaussian. The area depends on non-Gaussian distributions and, in particular, some conditional distributions are also non-Gaussian, but known as the Perks' functions: $\phi(x)=c\left(e^{x}+2 \cos \psi+e^{-x}\right)^{-1}, 0 \leqq \psi \leqq \pi / 2$, with the characteristic function $C(x, v)=(\pi / \psi) \sinh (\psi v) / \sinh (\pi v)$. (Received October 10, 1955.)

\section{TOPOLOGY}

\section{6t. R. H. Bing: A simple closed curve which pierces no disk.}

A simple closed curve $J$ is said to pierce a disk $D$ if $J$ links the boundary of $D$ and $J \cdot D$ is a single point. An example is given of a simple closed curve in $E^{3}$ which links no disk in $E^{3}$. Essential use is made of the result that if $M$ is a 2-manifold in $E^{3}$ and $f$ is a non-negative continuous function defined on $M$, then there is a 2-manifold $M^{\prime}$ and a homeomorphism $h$ of $M$ onto $M^{\prime}$ such that the $M^{\prime}$ is piecewise linear where $f h^{-1}$ is positive and the distance between $x$ and $h(x)$ is less than $f(x)$. (Received October 10, 1955.)

\section{R. H. Bing: Periodic homeomorphisms of $E^{3}$ which are not} orthogonal.

It is shown that for each integer $n$ there is a homeomorphism of period $n$ of $E^{3}$ onto itself such that the set of fixed points of the homeomorphism is a wild line. (Received October 10, 1955.)

\section{L. J. Heider: A lattice characterization of compact Hausdorff} continuous function spaces.

Let $L$ denote a lattice within which a sublattice $R$, lattice-isomorphic to the lattice of all real numbers, has been designated with element $r$ of $R$ corresponding to real number $\rho$. Describe as a real lattice homomorphism of $L$ every real-valued function $\phi$ defined on all of $L$ and such that: $\phi(f \bigvee g)=\phi(f) \bigvee \phi(g), \phi(f \wedge g)=\phi(f) \wedge \phi(g), \phi(r)=\rho$ for all $f, g$ in $L$ and all $r$ in $R$. In particular if $L=C_{0}^{*}(X)$ where $C_{0}^{*}(X)$ is a sublattice of the lattice $C^{*}(X)$ of all real-valued functions defined and bounded on a set $X$ and $C_{0}^{*}(X)$ includes all constant functions $r(x) \equiv \rho$, let these constant functions form the designated sublattice $R$. For such lattices the functions $\phi_{x}$ with $\phi_{x}(f)=f(x)$ determined by points $x$ in the set $X$ may be called point real lattice homomorphisms. One may now prove: a necessary and sufficient condition that an abstract lattice $L$ be latticeisomorphic to a lattice $C^{*}\left(X^{T}\right)$ of all real-valued continuous functions on a compact Hausdorff space $X^{T}$ is that $L$ contain a sublattice $R$ lattice-isomorphic to the real numbers such that if $X=\{x, y, \cdots\}$ be an index set for the set $\Phi=\left\{\phi_{x}, \phi_{y}, \cdots\right\}$ of all real lattice homomorphisms of $L$ as containing $R$ and $C^{*}(X)$ be the lattice of all real-valued bounded functions defined on $X$, then, under the definitions $f(x)=\phi_{x}(f)$, the elements $f$ of $L$ each determine distinct elements of $C^{*}(X)$ while every sublattice 
of $C^{*}(X)$ containing properly all functions thus determined has a real lattice homomorphism other than the point real lattice homomorphisms. (G. Birkhoff, Lattice theory, p. 176, problem 81.) (Received October 6, 1955.)

159. Melvin Henriksen (p) and J. R. Isbell: Completions of uniformisable spaces.

$X$ always denotes a uniformisable space, $C(X)$ the algebra of all continuous realvalued functions on $X$, and $S(X)$ a subalgebra with unit of $C(X)$ such that for every $x \in X$, and every neighborhood $U$ of $X$, there is an $f \in S(X)$ such that $f(x)=0$, $f(X-U)=1$. If $X$ has nonmeasurable character, then every completion of $X$ is a subspace $K(S)$ of the space of maximal ideals (in the Stone topology) of $S(X)$. Conversely, for any such $S(X), K(S)$ is the completion of $X$ in the coarsest structure making every element of $S(X)$ uniformly continuous. If, in addition, $f \in S(X)$ implies $|f| \in S(X)$, and $f \in S(X), f^{-1} \in C(X)$ implies $f^{-1} \in S(X)$, then $K(S)$ is $v X$, the completion of $X$ in the coarsest structure making every element of $C(X)$ uniformly continuous. Hence, if $X, Y$ are $Q$-spaces (i.e., complete in this structure), and $S(X), S(Y)$ satisfy the above hypotheses, and are isomorphic, then $X$ and $Y$ are homeomorphic. If $D(X)$ denotes the number of distinct completions of $X$, then $D(X) \geqq \max (|\beta X-v X|, 1)(\beta X=$ Stone-Čech compactification). If there is an unbounded $f \in C(X)$, then $D(X) \geqq \exp \exp$ so. If $X$ has non-measurable character, then $D(X) \leqq \exp |C(X)|$. (The authors were supported in part by the National Science Foundation.) (Received October 13, 1955.)

160. J. E. Kelley: Concerning a problem of Sikorski. Preliminary report.

Sikorski (Colloquium Mathematicum vol. 1 (1948)) announced that in spaces satisfying the Kuratowski axioms certain implications, and no others, hold in general between the following properties: $B$, second countability; $M(N)$ every well ordered increasing (decreasing) sequence of open sets is countable; $I$, every isolated set is countable; $D$, separability; $S$, every collection of mutually exclusive open sets is countable. Sikorski asked whether additional implications hold in general for normal spaces. Using results of Marczewski (Fund. Math. vol. 2 (1921) pp. 172-183) and of Alexandroff and Urysohn (Verhandelingen Der Koninklijke Akademie van Wetenschappen te Amsterdam, Eerste Sectie No. 1 (1929-1931)) one can construct normal spaces which are counter-examples for twelve of the eighteen possible additional implications. Every compact perfectly normal space has property $M$. (Received October 10, 1955.)

161t. I. S. Krule: On connected subsets of a continuum endowed with a struct.

If $X$ is a Hausdorff space and $L$ is a nonvoid, closed, transitive subset of $X \times X$, then $L$ is called a struct on $X$. (For other terminology see Bull. Amer. Math. Soc. Abstracts 61-1-123 (the author) and 61-1-127 (A. D. Wallace).) If $L$ is a struct on $X$, let $K_{L}$ be the set of all $L$-minimal elements and let $G_{L}=\{x \in X: L(x)=X\}$. THEOREM: If $L$ is a continuous struct on the continuum $X$ and if $K_{L}$ is a proper connected subset of $X$, then no closed subset of $G_{L}$ cuts $X$. (Cf. R. J. Koch and A. D. Wallace, Maximal ideals in compact semigroups, Duke Math. J. vol. 21 (1954) pp. 681-685, LEMMA 2.) THEOREM: If $L$ is a monotone continuous struct on the continuum $X$, if $L(X)=X$, and if $J$ is a maximal proper $L$-ideal containing $K_{L}$, then no subset of $X-J$ cuts $X$. The 
conclusion of this theorem does not hold if $L$ is not monotone even though $L$ may be reflexive. As a corollary to this theorem there is the following theorem of Wallace (Struct ideals, Proc. Amer. Math. Soc. vol. 6 (1953) pp. 634-638, THEOREM 5): $L e t ~ L$ be a continuous monotone struct on the continuum $X$. Suppose that $a \in X-K_{L}$ and let $C$ be the component of $X-L_{a}$ containing $K_{L}$. Then $L_{a}=a \cup\left(C^{*}-C\right)$ and if $a$ is $L$ reflexive, then $a \in C^{*}-C$. (Received October 11, 1955.)

\section{2t. G. O. Sabidussi: On a multiplication of graphs.}

Let $\left\{X_{\alpha}\right\}, \alpha \in A$, be an indexed set of graphs $X_{\alpha}$ given by $V\left(X_{\alpha}\right)$ and $E\left(X_{\alpha}\right)$, the sets of vertices and edges of $X_{\alpha}$. By the product $\prod_{0 \alpha \in A} X_{\alpha}$ is meant the following graph $Z: V(Z)=\prod_{\alpha \in A} V\left(X_{\alpha}\right)$ (Cartesian product); $[P, Q] \in E(Z)(P, Q \in V(Z))$ if, for exactly one $\alpha_{0} \in A,\left[P_{\alpha_{0}}, Q_{\alpha_{0}}\right] \in E\left(X_{\alpha_{0}}\right)$ and $P_{\alpha}=Q_{\alpha}$ otherwise (here $P_{\alpha}, Q_{\alpha}$ denote the $\alpha$ th component of $P, Q$ respectively). It is shown that for all $\aleph_{n}$ this multiplication together with graph addition turns the set $X \aleph_{n}$ of all nonisomorphic graphs $X$ with cardinal $V(X)<s_{n}$ into a commutative semiring with zero and unit elements. This semiring $\mathfrak{X}_{\mathfrak{N} n}$ does not possess a Euclidean algorithm; however, the decomposition of graphs $\in \mathfrak{X}_{\mathfrak{N} n}$ into prime factors is unique. This implies that $\mathfrak{X}_{\mathfrak{N} n}$ is a cancellation semigroup under graph multiplication. As an application of the unique prime factor decomposition it is shown that the automorphism group of a graph $X$ is isomorphic to the automorphism group of the graph $\sum X_{\alpha}$ where the $X_{\alpha}$ are the prime factors of $X$. (Received October 10,1955.)

J. W. T. Youngs, Associate Secretary 